ORIGINAL ARTICLE

\title{
Isoniazid pharmacokinetics in children treated for respiratory tuberculosis
}

\author{
H S Schaaf, D P Parkin, H I Seifart, C J Werely, P B Hesseling, P D van Helden, J S Maritz, \\ P R Donald
}

Arch Dis Child 2005;90:614-618. doi: 10.1136/adc.2004.052175

See end of article for authors' affiliations

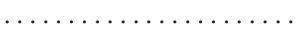

Correspondence to: Dr H S Schaaf, Paediatrics and Child Health, Stellenbosch University, PO Box 19063, 7505 Tygerberg, South Africa; hss@sun.ac.za

Accepted 9 August 2004
Aims: To define the pharmacokinetics of isoniazid $(\mathrm{INH})$ in children with tuberculosis in relation to the N-acetyltransferase 2 (NAT2) genotype.

Methods: The first order elimination rate constant $(k)$ and area under the concentration curve (AUC) were calculated in 64 children $<13$ years of age (median 3.8) with respiratory tuberculosis from $\mathbb{N H}$ concentrations determined $2-5$ hours after a $10 \mathrm{mg} / \mathrm{kg}$ INH dose. The NAT2 genotype was determined; 25 children were classified as homozygous slow (SS), 24 as heterozygous fast (FS), and 15 as homozygous fast (FF) acetylators.

Results: The mean (SD) $k$ values of the genotypes differed significantly from one another: SS 0.254 (0.046), FS 0.513 (0.074), FF 0.653 (0.117). Within each genotype a median regression of $k$ on age showed a significant decrease in $k$ with age. The mean (SD) INH concentrations (mg/l) two hours after INH administration were SS 8.599 (1.974), FS 5.131 (1.864), and FF 3.938 (1.754). A within genotype regression of 2 -hour $\mathbb{I N H}$ concentrations on age showed a significant increase with age. A within genotype regression of 3-hour, 4-hour, and 5-hour concentrations on age also showed a significant increase with age in each instance. In ethnically similar adults, mean (SD) 2-hour INH concentrations (mg/l) for each genotype were significantly higher than the children's: SS 10.942 (1.740), FS 8.702 (1.841), and FF 6.031 (1.431).

Conclusions: Younger children eliminate INH faster than older children and, as a group, faster than adults, and require a higher $\mathrm{mg} / \mathrm{kg}$ body weight INH dose to achieve serum concentrations comparable to adults.
M ore than 50 years after its introduction as an antituberculosis agent, ${ }^{1}$ isoniazid (INH) continues to form the cornerstone of all "first line" antituberculosis regimens and remains the only agent recommended for tuberculosis chemoprophylaxis in children. ${ }^{23}$ INH is most valued for its powerful bactericidal effect against the metabolically active organisms most commonly encountered in the sputum of adults with cavitating pulmonary tubercu$\operatorname{losis}^{4}$ and is the most valuable agent for preventing the development of resistance in companion agents. ${ }^{5}$ There is also evidence that INH suppresses the growth of non-multiplying organisms and that prolonged exposure of such organisms to concentrations above the minimal inhibitory concentration (MIC) leads to bacteriolysis. ${ }^{67}$

INH is well absorbed from the gastrointestinal tract, but is subject to significant first pass metabolism that may impact on its systemic concentrations. INH does not bind appreciably to plasma proteins, crosses membranes readily, and distributes into a compartment that approximates the total body water. ${ }^{8}$ Very little of the parent compound is excreted unchanged in the urine and the greater proportion is acetylated in the liver and the small intestine to acetylisoniazid prior to excretion in the urine. Acetylation capacity in any individual is genetically determined, and although the initial studies of INH pharmacokinetics seemed to indicate that INH was bimodally eliminated, ${ }^{9}$ there was the suspicion that its elimination was in fact trimodal. ${ }^{10}$ Modern molecular biology techniques and improved analytical methods have now established, beyond doubt, that INH is eliminated in accordance with a trimodal distribution of subtypes, fast (FF), intermediate (FS), and slow (SS), the fast (F) and slow (S) alleles being co-dominant. ${ }^{11}$

The pharmacokinetic characteristics of INH have been extensively studied in adults, ${ }^{8}$ but data in respect of children, and especially younger children are limited. Where such data are available cognisance has not been taken of the genotype or of the trimodality of INH elimination. ${ }^{12-14}$

This study was undertaken to improve our understanding of INH pharmacokinetics in children being treated for tuberculosis, making use of improved analytical technology and advances in our understanding of the polymorphisms governing INH metabolism.

\section{PATIENTS AND METHODS}

The study was undertaken in the Western Cape Province of South Africa, an area with a particularly high incidence of tuberculosis $(>600 / 100000$ population at the time of this study). Children less than 13 years of age with primary respiratory tuberculosis and some with abdominal tuberculosis were included in the study following informed written consent of the parent or legal guardian. If not already hospitalised for other reasons the children enrolled in the study were temporarily admitted to hospital on the morning of the study.

The age and weight of each child were recorded and the extent of pulmonary involvement and the presence of extrapulmonary tuberculosis noted. Children who were severely ill were excluded from the study. With appropriate counselling and written informed consent the human immunodeficiency virus (HIV) status of the children was assessed.

The INH used for the study was standard pharmaceutical grade in powder form obtained from Fluka Chemie AG (Buchs, Switzerland). The INH was accurately weighed to

\footnotetext{
Abbreviations: AUC, area under the concentration curve; FF, homozygous fast acetylator; FS, heterozygous fast acetylator; $\mathbb{N H}$, isoniazid; NAT2, N-acetyltransferase 2; SS, homozygous slow acetylator
} 
Table 1 Clinical features and special investigation results of the study children

\begin{tabular}{|c|c|c|}
\hline $\begin{array}{l}\text { Clinical feature or special } \\
\text { investigation }\end{array}$ & $\begin{array}{l}\text { Number } \\
n=64\end{array}$ & $\%$ \\
\hline \multicolumn{3}{|l|}{ Age group } \\
\hline $0-2$ years & 18 & 28 \\
\hline$>2-5$ years & 24 & 38 \\
\hline$>5-13$ years & 22 & 34 \\
\hline Weight loss & 50 & 78 \\
\hline Weight < 3rd centile & 31 & 48 \\
\hline Cough $>2$ weeks & 39 & 61 \\
\hline Household TB contact & 35 & 55 \\
\hline \multicolumn{3}{|l|}{ Mantoux test } \\
\hline $0-4 \mathrm{~mm}$ & $13(7=\mathrm{HIV}$ infected $)$ & 20 \\
\hline $5-14 \mathrm{~mm}$ & 6 & 9 \\
\hline$\geqslant 15 \mathrm{~mm}$ & $41(6=\mathrm{HIV}$ infected $)$ & 64 \\
\hline Not done & 4 & 6 \\
\hline HIV infected & 13 & 20 \\
\hline \multicolumn{3}{|l|}{ Chest radiograph } \\
\hline Lymphadenopathy & 44 & 69 \\
\hline Collapse/opacification & 33 & 52 \\
\hline Pleural effusion & 14 & 22 \\
\hline Miliary & 8 & 13 \\
\hline \multicolumn{3}{|l|}{ Extra-pulmonary TB } \\
\hline Peripheral lymph nodes & 14 & 22 \\
\hline Pleural effusion & 14 & 22 \\
\hline Miliary & 8 & 13 \\
\hline TB meningitis stage 1 & 4 & 6 \\
\hline Pericardial effusion & 1 & 2 \\
\hline $\begin{array}{l}\text { Culture or histology confirmed } \\
\text { tuberculosis }\end{array}$ & 41 & 64 \\
\hline
\end{tabular}

give a dose of $10 \mathrm{mg} / \mathrm{kg}$ according to the child's weight the previous day. The INH powder was dissolved in $5-10 \mathrm{ml}$ of water and administered by one of the study personnel orally with a syringe or, in the case of very young children, through a nasogastric tube and washed down with water. A light breakfast was permitted 60-90 minutes later.

Four blood specimens of $1-1.5 \mathrm{ml}$ each, taken at 2, 3, 4, and 5 hours post-dose, were collected in ethylenediaminetetraacetate (EDTA) coated tubes, chilled, and delivered on ice to the laboratory within one hour of taking the last specimen. INH concentrations were determined by the high performance liquid chromatography (HPLC) method of Seifart and colleagues. ${ }^{15} \mathrm{~A}$ further single $3 \mathrm{ml}$ sample was collected into an EDTA coated tube for DNA analysis.

\section{NAT2 genotyping}

Genomic DNA (gDNA) was prepared via the salting out procedure of Miller and colleagues. ${ }^{16}$ This gDNA was subsequently analysed for the NAT2*5, ${ }^{*} 6,2 * 7,2 * 12,2 * 13$, and $2^{*} 14$ alleles, ${ }^{17}$ via a polymerase chain reaction (PCR) based strategy, as previously described. ${ }^{11}$ Separate PCR aliquots were restricted with the MspI, FokI, KpnI, TaqI, DdeI, and BamHI restriction enzymes (according to the manufacturer's recommendations) to delineate the polymorphisms at nucleotide positions 191, 282, 481, 590, 803, and 857, respectively. According to the Vatsis nomenclature the wild type fast allele (F) is assigned as NAT2* $4,2^{*} 12$, or $2{ }^{*} 13 .{ }^{17}$ These alleles confer normal enzyme activity on the NAT2 protein, while the mutant slow alleles (S), classified as NAT2 ${ }^{*}, 2{ }^{*} 6,2{ }^{*} 7$, and $2{ }^{*} 14$ in humans, confer a decreased enzyme activity on the NAT2 protein.

The T341C mutation of the NAT2*5 allele was typed via an allele specific PCR protocol, employing the confronting primer PCR method of Hamajima and colleagues. ${ }^{18}$ In this case the standard PCR mixture contained two primer sets, primer set I [5'-876nt TTAGAGGCTATTTTTGAT CACA ${ }^{897 \mathrm{nt}}-3^{\prime}$ and $5^{\prime}{ }^{1081 n t}$ ATGTAATTCCTGCCGTCAG $\left.{ }^{1063 n t}-3^{\prime}\right]$, which initiates amplification in the case of the $341 \mathrm{C}$ allele (a 187bp product), and primer set II $\left[5^{\prime}{ }_{-}{ }^{1045 n t}\right.$ TTCTCCTGCAGGTGAC

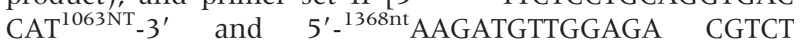
$\left.\mathrm{GC}^{1349 \mathrm{nt}}-3^{\prime}\right]$, which only amplifies in the case of the 341T allelic sequence (a $323 \mathrm{bp}$ product). In addition, outermost primers [ [ ${ }^{876 n t}$ TTAGAG GCTATTTTTGATCACA ${ }^{897 n t}$ and ${ }^{1368 n t}$ AAGATGTTGGAGACGTCTGC ${ }^{\text {1349nt }}$ ] also amplify a gene specific PCR product (475bp) in the reaction mixture which serves as an internal amplification control.

The KpnI, BamHI, and MspI generated profiles were subsequently also cleaved with PstI, to facilitate the analyses of these DNA profiles. This second cleavage generated a profile of bands less than $500 \mathrm{bp}$ in size which enabled their resolution via $5 \%$ polyacrylamide gel electrophoresis (PAGE), using the Mini-Protean II gel system (Bio-Rad Laboratories, USA); DNA bands were visualised by silver staining, as described by Bassam and colleagues. ${ }^{19}$ The PstI restriction sites occur at nucleotide positions 1050 and 1347 of the NAT2 gene sequence (GenBank accession number D10870); we have found these sites to be conserved in all samples that we have analysed thus far (more than 700). The use of this second enzymatic restriction improves the sensitivity of analysis of the polymorphisms at 481, 857, and 191 respectively.

\section{Pharmacokinetic parameters}

The apparent first order elimination rate constants $\left(k, h^{-1}\right)$ of the individual patients were calculated from the linear regression of $\ln \left(C_{t}\right)$ and $t$, where $\ln \left(C_{t}\right)$ is the natural logarithm of the concentration at time t; observations of $\mathrm{C}_{t}$ were made at 2, 3, 4, and 5 hours after oral administration of $10 \mathrm{mg} / \mathrm{kg}$ body weight of INH. The area under the curve (AUC) over the interval 2-5 hours after the dose was calculated by integration of the AUC of each of the constituent subintervals $(2-3,3-4$, and $4-5$ hours after the dose) by standard methods. ${ }^{20}$

\section{Statistical methods}

Tests of homogeneity of group means were performed by one way analysis of variance. The general linear model approach was used for data subject to more than one classification criterion. The association between quantitative variables was examined by regression; in the case of the relation between age and $k$ values the heteroscedasticity of the data required

Table 2 The mean first order elimination rate constant (k), the area under the curve (AUC) during the period 2-5 hours after dosing, and the mean serum INH concentrations at 2, 3, 4, and 5 hours after dosing with $10 \mathrm{mg} / \mathrm{kg}$ isoniazid (INH)

\begin{tabular}{|c|c|c|c|c|c|c|}
\hline \multirow[b]{2}{*}{ Genotype (n) } & \multirow[b]{2}{*}{$K(S D)\left(h^{-1}\right)$} & \multirow[b]{2}{*}{ AUC (SD) (mg/l/h) } & \multicolumn{4}{|c|}{ Mean INH concentration (SD) (mg/l) } \\
\hline & & & $2 \mathrm{~h}$ after dosing & $3 \mathrm{~h}$ after dosing & $4 \mathrm{~h}$ after dosing & $5 \mathrm{~h}$ after dosing \\
\hline SS (25) & $0.254(0.046)$ & $18.356(4.692)$ & 8.599 (1.974) & $6.585(1.615)$ & $5.099(1.355)$ & $4.007(1.179)$ \\
\hline FS (24) & $0.513(0.074)$ & $8.246(3.349)$ & $5.131(1.864)$ & 3.168 (1.294) & $1.955(0.885)$ & $1.178(0.565)$ \\
\hline FF (15) & $0.653(0.117)$ & $5.371(3.076)$ & $3.938(1.754)$ & $2.045(1.060)$ & $1.125(0.646)]$ & $0.642(0.426)$ \\
\hline$F(d f, d f)$ & $88.92(2.61)$ & $58.42(2.55)$ & $33.39(2.59)$ & $61.94(2.61)$ & $84.91(2.61)$ & $0.642(2.57)$ \\
\hline p value & $<0.0005$ & $<0.0005$ & $<0.0005$ & $<0.0005$ & $<0.0005$ & $\begin{array}{l}<0.0005 \\
\end{array}$ \\
\hline
\end{tabular}

SD, standard deviation; SS, homozygous slow acetylators; FS, heterozygous fast acetylators; FF, homozygous fast acetylators. 
Table 3 Significance of a straight line regression of $\mathbb{N N H}$ concentrations on age with a common slope for genotype

\begin{tabular}{|c|c|c|c|c|c|}
\hline \multirow{2}{*}{$\begin{array}{l}\text { Time after INH } \\
\text { dosing (hours) }\end{array}$} & \multicolumn{3}{|c|}{ Ordinates at mean age } & \multirow[b]{2}{*}{ Slope } & \multirow[b]{2}{*}{$\mathbf{p}^{*}$} \\
\hline & SS & FS & FF & & \\
\hline 2 & 8.622 & 5.078 & 3.882 & 0.278 & $<0.0005$ \\
\hline 3 & 6.650 & 3.128 & 2.001 & 0.214 & $<0.0005$ \\
\hline 4 & 5.150 & 1.924 & 1.092 & 0.166 & $<0.0005$ \\
\hline 5 & 4.048 & 1.129 & 0.604 & 0.136 & $<0.0005$ \\
\hline
\end{tabular}

the use of median regression. This is essentially robust regression that entails the fitting of parallel straight lines to the three groups of data. ${ }^{21}$

The Institutional Review Board of the Faculty of Health Sciences, Stellenbosch University, approved the study. The parents or legal guardians of all of the children gave written informed consent for their children's participation in the study.

\section{RESULTS}

Sixty four children with a median age of 3.8 years (lower quartile 1.8 years, upper quartile 7.8 years) were included in the study. Table 1 summarises details of age and radiological and clinical features. Although 50 (78\%) children had experienced recent weight loss, confirmed by reference to a "Road to Health" card, only 31 (48\%) had a weight for age of less than 3rd centile (National Center for Health Statistics, USA). Thirteen (20\%) children were HIV infected and 15 $(23 \%)$ children suffered from abdominal tuberculosis. In 41 children $(64 \%)$ tuberculosis was confirmed by isolation of $M$ tuberculosis from one or more clinical specimens or identification of acid fast bacilli following tissue biopsy.

Twenty five children (39\%) with median age of 2.8 years were genotyped as homozygous slow acetylators of INH, 24 $(38 \%)$ with median age of 3.9 years heterozygous fast acetylators, and $15(23 \%)$ with median age of 4.1 years as homozygous fast acetylators. With respect to the variable age, the differences between the genotype groups are not statistically significant.

Table 2 presents the mean first order elimination rate constant $(k)$, the mean area under the concentration versus time curve (AUC) for the period 2-5 hours after dosing, and the mean INH concentration at 2, 3, 4, and 5 hours after administration of INH. The means of each of the variables differed significantly between genotypes $(p<0.0005$ in each instance). In the case of the variable $k$ the group variance increases with group means and for formal testing of homogeneity of group means the $k$ values were transformed to logarithms; this had the effect of stabilising the variances. The ratio of the largest to the smallest variance gave observed $\mathrm{F}(25.22)=1.49$; this conservative test gives $\mathrm{p}=0.18$. A formal test of normality of residuals was not performed but dotplots indicated no obvious departure from normality. Although one way ANOVA is sensitive to lack of homoscedasticity it is robust against departures from normality. A Kruskal-Wallis test applied to $k$ (or its logarithms) results in a similar $\chi^{2}$ (49.17; degrees of freedom $\left.=2, p<0.0005\right)$. Similar checks of the results relating to other variables in table 2, confirmed the statistical significance statements. Confidence limits (95\%) for the location of the $\log$ transformed data are: SS $(0.234$ to 0.268$)$, FS (0.477 to 0.538 ), FF (5.93 to 0.700 ). They are clearly well separated.

At the extremes the two hour INH concentrations varied from a high of $12 \mathrm{mg} / \mathrm{l}$ in a homozygous slow acetylator to a low of $<2 \mathrm{mg} / \mathrm{l}$ in a homozygous fast acetylator. Within each genotype the means of $k$, AUC, and 2-hour, 3-hour, 4-hour, and 5-hour INH concentrations were not significantly associated with either HIV infection or the presence of abdominal tuberculosis.

With regard to the relation between age and $k$ a regression of median values, taking into account heteroscedasticity of the data, confirms a significant decline in $k$ with increasing age for each genotype. The results of fitting straight line regressions, $k$ on age, with common slope parameter, were: slope $=-0.00521$, standard error 0.00114 , giving $\mathrm{p}<0.001 .^{21}$ The fitted median values at the common mean age are 0.248 , $0.500,0.620$ respectively for the three genotypes; they differ significantly from each other according to a global test $(\mathrm{p}<0.0005)$, and the two values closest to each other, FS and FF also differ significantly. ${ }^{21}$ Table 3 summarises the result of fitting straight line regressions, INH concentration at each of the time points, 2-hours, 3-hours, 4-hours, and 5 -hours on age with a common slope for the genotypes, and confirms a significant rise in each of the 2-hour, 3-hour, 4-hour, and 5-hour INH concentrations with age $(p<0.0005$ in each instance).

In order to explore the relation of INH elimination and age further we compared the findings in this group of children with those obtained by our research group in a similar ethnic population of adults who were also genotyped and phenotyped after receiving $5 \mathrm{mg} / \mathrm{kg}$ and $10 \mathrm{mg} / \mathrm{kg}$ doses of INH. ${ }^{11}$ Among the adult patients INH concentrations were

Table 4 Adult patients $(n=60)$ : the mean first order elimination rate constant $(k)$, the area under the curve (AUC) during the period 2-5 hours, the mean serum concentrations at 2 and 3 hours after dosing with $10 \mathrm{mg} / \mathrm{kg}$, and the calculated INH concentrations 4 and 5 hours after dosing

\begin{tabular}{|c|c|c|c|c|c|c|}
\hline \multirow[b]{2}{*}{ Genotype } & \multirow[b]{2}{*}{$k(S D)\left(h^{-1}\right)$} & \multirow[b]{2}{*}{ AUC (SD) (mg/l/h) } & \multicolumn{4}{|c|}{ Mean INH concentration (SD) (mg/l) } \\
\hline & & & $2 \mathrm{~h}$ after dosing & $3 \mathrm{~h}$ after dosing & $4 \mathrm{~h}$ after dosing & $5 \mathrm{~h}$ after dosing \\
\hline SS & $0.193(0.026)$ & 24.870 (4.077) & $10.942(1.740)$ & $8.943(1.448)$ & $7.433(1.268)$ & $6.169(1.141)$ \\
\hline FS & $0.430(0.080)$ & $15.338(4.018)$ & $8.702(1.841)$ & $5.789(1.446)$ & $3.991(1.225)$ & $2.631(1.015)$ \\
\hline $\mathrm{FF}$ & $0.678(0.056)$ & $8.139(2.167)$ & 6.031 (1.431) & $3.043(0.896)$ & $1.748(0.510)$ & $0.877(0.257)$ \\
\hline
\end{tabular}

SD, standard deviation; SS, homozygous slow acetylators; FS, heterozygous fast acetylators; FF, homozygous fast acetylators. 


\begin{tabular}{llrl|}
\hline \multicolumn{3}{l}{ Table 5} & \multicolumn{3}{l}{ Results of the ANOVA of variable $k$} \\
\hline Factor & df & F & P \\
\hline Genotype & 2,118 & 263.09 & $<0.0005$ \\
Adult/child & 1,118 & 6.79 & 0.010 \\
Gen*adult/child & 2,118 & 3.96 & 0.022 \\
\hline
\end{tabular}

determined at $0.5,1,2,3,4.5$, and 6 hours after dosing. Table 4 presents the adults' mean INH concentrations at 2 and 3 hours after dosing, together with the calculated 4- and 5 -hour INH concentrations, $k$ values, and AUC for the period $2-5$ hours after dosing. These means may be compared with the paediatric data in table 2. However, for a more formal comparison of the two data sets we recall the basic model relating concentration and time of observation according to which $\log$ (concentration) is a linear function of time. The slope coefficient is the rate constant $\mathrm{k}$, and the implication is that the data of any individual can be summarised in this constant and the intercept of the linear function. Equivalently the data can be summarised in $k$ and the fitted ordinate at any chosen time. For this analysis we use the ordinate at 2 hours, and for convenience refer to it as A.

Genotype and adult/child status are treated as factors with three and two levels, respectively, in a two way analysis of variance to test for the significance of these factors. Table 5 shows the results of the ANOVA of variable $k$.

All effects are clearly highly significant. As a check a rank regression analysis was performed, as implemented in MINITAB, and essentially the same results were obtained. Inspection of tables 2 and 4 shows that the significant interaction is explained by the mean values of $k$ being different between children and adults at genotypes SS and FS, but not at FF. To confirm, the Bonferroni confidence intervals with global confidence coefficient 0.95 for the differences between the adult and child means are: SS (0.0029 to 0.1201$)$, FS $(0.0274$ to 0.1386$)$, FF $(-0.1017$ to 0.0517). According to the ANOVA the overall means of the genotypes differ significantly. Bonferroni intervals, as above, for the differences are, SS-FS $(-0.2826$ to -0.2020$)$, SS-FF $(-0.4855$ to -0.3895$)$, FS-FF $(-0.2423$ to -0.1481$)$.

Table 6 present the results of an analysis of variance of variable $\mathrm{A}$. The genotype and adult/child main effects are clearly significant. These results have also been confirmed by a rank regression analysis. The Bonferroni confidence intervals with global confidence coefficient 0.95 for the genotype mean differences are: SS-FS (1.628 to 3.402), SSFF (3.668 to 5.782), FS-FF ( 1.172 to 3.248 ). The conclusion is that average levels of concentration differ between adults and children, and also between genotypes.

Finally, as further confirmation of the significance of the genotype and adult/child differences a multivariate ANOVA was performed with the joint response variables $k$ and A. For the test of the main effect of factor adult/child the Wilk F-statistic is $F(2,117)=31.10, p<0.0005$; for the test of the main effect of factor genotype the Wilk F-statistic is $\mathrm{F}(4,234)=80.28, \mathrm{p}<0.0005$; and for the interaction it is $\mathrm{F}(4,234)=2.40, \mathrm{p}=0.051$.

After transforming the children's weight for age to $z$ scores, analysis of variance did not reveal any significant association of the pharmacokinetic parameters with body weight.

\section{DISCUSSION}

The results of this study illustrate once again the considerable differences in exposure to INH that exist between homozygous slow acetylators of INH and the heterozygous and homozygous fast acetylators, and show that these differences
Table 6 Results of the ANOVA of variable A

\begin{tabular}{llll}
\hline Factor & df & F & P \\
\hline Genotype & 2,118 & 62.68 & $<0.0005$ \\
Adult/child & 1,118 & 62.51 & $<0.0005$ \\
Gen*adult/child & 2,118 & 1.68 & 0.191 \\
\hline
\end{tabular}

also exist in children. Our data also confirm that younger children eliminate INH faster than older children and in a trimodal model of INH elimination there is a significant age related decline in the first order elimination rate constant $(k$, $\mathrm{h}^{-1}$ ) with age in all three genotypes. Furthermore the exposure of the children to INH, as reflected by the first order elimination rate constant, AUC for the period 2-5 hours after dosing, and INH concentrations at different time intervals after dosing, is significantly less than that of a group of adults drawn from the same population and receiving the same $\mathrm{mg} / \mathrm{kg}$ body weight dose of INH. These findings, taking into account the NAT2 genotype for the first time, confirm the suggestions of earlier workers, based on phenotypification, that younger children eliminate INH faster than older children, and children, as a group, faster than adults. ${ }^{22-25}$ The significantly faster elimination of INH by infants and younger children has been ascribed to the relatively greater mass of the liver in proportion to total body weight and it has been proposed that more optimal doses would be calculated on the basis of body surface area rather than body weight. Given that INH will most often be used in developing countries under programme conditions it is unlikely that this will be possible.

The normal range of INH concentrations two hours after dosing has been given as $3-5 \mathrm{mg} / \mathrm{l}^{26}$ alternatively it has been suggested that a 3-hour concentration of $1.5 \mathrm{mg} / \mathrm{l}$ is desirable. $^{27}$ It is therefore noteworthy that seven $(35 \%)$ of the homozygous fast acetylators had a 2-hour INH concentration of less than $3 \mathrm{mg} / \mathrm{l}$ and nine (45\%) did not reach a 3-hour post-dose concentration of $1.5 \mathrm{mg} / \mathrm{l}$. It is inevitable that using a lower dose of $5 \mathrm{mg} / \mathrm{kg}$ body weight will lead to an even greater proportion of homozygous fast acetylators and probably a significant proportion of heterozygote fast acetylators failing to achieve the recommended concentrations.

The above findings provide justification for the official recommendations of some professional bodies that children should receive higher $\mathrm{mg} / \mathrm{kg}$ doses of INH than adults. Thus while the International Union Against Tuberculosis and Lung Disease, ${ }^{28} 29$ the World Health Organisation, ${ }^{30}$ and the Joint Tuberculosis Committee of the British Thoracic Society ${ }^{31}$ recommend an INH dosage of $4-6 \mathrm{mg} / \mathrm{kg}$ body weight, the American Academy of Pediatrics recommends an INH dose of $10-15 \mathrm{mg} / \mathrm{kg}$ body weight. ${ }^{2}$ In debating the most appropriate dose of INH for use in children, some caution is necessary. Many of the studies documenting a satisfactory clinical response to an INH dose of $5 \mathrm{mg} / \mathrm{kg}$ emanate from populations with a predominance of homozygous slow acetylators; the relative therapeutic disadvantage of the heterozygote fast acetylator will also be concealed by the synergistic potential of a multidrug regimen. Under adverse circumstances, however, such as failure of full compliance, compromised absorption of INH itself, or its companion drugs, in particular rifampicin, ${ }^{32}$ or the sequestration of organisms in poorly perfused lesions, ${ }^{33-35}$ this relative disadvantage may be exposed. In the light of our findings, and in agreement with the recommendations of the American Academy of Pediatrics, we suggest that young children less than 5 years of age should receive an INH dose of at least $10 \mathrm{mg} / \mathrm{kg}$ to ensure that the faster acetylators of INH are exposed to adequate serum concentrations of INH. 


\section{Authors' affiliations}

H S Schaaf, P B Hesseling, P R Donald, Department of Paediatrics and Child Health, Faculty of Health Sciences, Stellenbosch University and Tygerberg Children's Hospital, South Africa

D P Parkin, H I Seifart, Department of Pharmacology, Faculty of Health Sciences, Stellenbosch University, South Africa

C J Werely, P D van Helden, Department of Medical Biochemistry, Faculty of Health Sciences, Stellenbosch University, South Africa J S Maritz, Biostatistics Unit of the South African Medical Research Council, South Africa

Financial assistance: Harry and Doris Crossley Foundation (HS Schaaf) and National Research Foundation (PD van Helden)

Competing interests: none declared

\section{REFERENCES}

1 Fox HH. The chemical approach to the control of tuberculosis. Science 1952;1 16:129-34.

2 American Academy of Pediatrics. Tuberculosis. In: Pickering LK, ed. Red Book: 2003 Report of the Committee on Infectious Diseases, 26th edn. Elk Grove Village, IL: American Academy of Pediatrics, 2003:642-60.

3 Centers for Disease Control and Prevention. Targeted tuberculin testing and treatment of latent tuberculosis infection. MMWR 2000;49:1-51

4 Donald PR, Sirgel FA, Botha FJ, et al. The early bactericidal activity of isoniazid related to its dose size in pulmonary tuberculosis. Am J Respir Crit Care Med 1997; 156:895-900.

5 Mitchison DA. Role of individual drugs in the chemotherapy of tuberculosis Int J Tuberc Lung Dis 2000;4:796-806.

6 Crowle AJ, Sbarbaro JA, May MH. Effects of isoniazid and ceforamide against virulent tubercle bacilli in cultured human macrophages. Tubercle 1988;69:15-25

7 McCune RM, Feldman FM, Lambert HP, et al. Microbial persistence 1. The capacity of tubercle bacilli to survive in mouse tissues. J Exp Med 1966;123:445-68.

8 Weber WW, Hein HW. Clinical pharmacokinetics of isoniazid. Clin Pharmacokinet 1979;4:401-22

9 Evans DA, Manley KA, McKusick VA. Genetic control of isoniazid metabolism in man. BMJ 1960;2:485-91.

10 Sunahara S, Urano M, Agawa M. Genetic and geographical studies on isoniazid inactivation. Science 1961;134:1530-1.

11 Parkin DP, Vandenplas S, Botha FJH, et al. Trimodality of isoniazid elimination. Am J Respir Crit Care Med 1997;155:1717-22.

12 Pariente-Khayat A, Rey E, Gendrel D, et al. Isoniazid acetylation metabolic ratio during maturation in children. Clin Pharmacol Ther 1997;62:377-83.

13 Rey E, Gendrel D, Treyluyer JM, et al. Isoniazid pharmacokinetics in children according to acetylator phenotype. Fund Clin Pharmacol 2001; 15:355-9.

14 Seth V, Beotra A. Antituberculosis drugs. II: Clinical pharmacokinetics in Indian children. In: Seth V, eds. Essentials of tuberculosis in children. Delhi, India: Jaypee Brothers Medical Publishers, 1997:291-303.
15 Seifart HI, Gent WL, Parkin DP, et al. High-performance liquid chromatographic determination of isoniazid, acetylisoniazid and hydrazine in biological fluids. J Chromatograph B 1995;674:269-75.

16 Miller SA, Dykes DD, Polesky HF. A simple salting out procedure for extracting DNA from human nucleated cells. Nucleic Acids Res 1988;16:1215.

17 Vatsis KP, Weber WW, Bell DA, et al. Nomenclature for N-acetyltransferases. Pharmacogenetics 1995;5:1-17.

18 Hamajima NT, Saito T, Matsuo K, et al. Polymerase chain reaction with confronting two-pair primers for polymorphism genotyping. Jpn J Cancer Res 2000;91:865-8.

19 Bassam BJ, Caetano-Anollés G, Gresshoff PM. Fast and sensitive silver staining of DNA in polyacrylamide gels. Anal Biochem 1991;196:80-3.

20 Weber WW. Determination of the human acetylator status. In: The acetylator genes and drug response. New York: Oxford University Press, 1987:151-73.

21 Maritz JS, Donald PR, Schaaf HS, et al. The association of age and the elimination of isoniazid: an example of median regression with heteroscedasticity. S Afr Statist J 2003;37:191-202.

22 Houin G. Tillement JP. Consequences thérapeutiques de la mesure de l'indice $\mathrm{d}^{\prime}$ inactivation de l'isoniazide au cours du traitement antituberculeux. Therapie 1980;35:597-605.

23 Advenier C, Saint-Aubin A, Scheinmann P, et al. Pharmacocinétique de l'isoniazide chez l'enfant. Rev Fr Mal Respir 1981:9:365-74.

24 Bouveret JP, Hanoteau J, Gerbeaux J, et al. Variations de l'indice $\mathrm{d}^{\prime}$ inactivation de l'isoniazide au cours des traitements antituberculeux chez l'enfant. Archiv Fr Pediatr 1983;40:615-19.

25 Lupasco I, Algeorge G, Ghiu-Cimpeanu V. Taux d'isoniazide actif dans le serum des enfants tuberculeux. Pneumon Coeur 1965:21:1105-14.

26 Peloquin CA. Therapeutic drug monitoring: principles and application in mycobacterial infections. Drug Ther 1992;22:31-6.

27 Vivien JN, Thibier R, Grosset J, et al. Resultats précoces de l'isoniazidothérapie en fonction du taux d'isoniazide actif dans le sérum. Rev Tuberc 1958;22:208-22.

28 International Union Against Tuberculosis and Lung Disease. Tuberculosis in children: guidelines for diagnosis, prevention and treatment. Bull Int Union Tuberc Lung Dis 1991;66:61-7.

29 Enarson DA, Rieder HL, Arnodottir T, et al. Management of tuberculosis: a guide for low income countries, 5th edn. Paris: IUATLD, 2000

30 World Health Organisation. Standardized treatment regimen. In: Treatment of tuberculosis: guidelines for national programmes. WHO/CDS/ TB2003.313. Geneva: WHO, 2003:27-38.

31 Joint Tuberculosis Committee of the British Thoracic Society. Chemotherapy and management of tuberculosis in the United Kingdom: recommendations 1988. Thorax 1998;53:536-48.

32 Sahai J, Gallicano K, Swick L, et al. Reduced plasma concentrations of antituberculosis drugs in patients with HIV infection. Ann Intern Med 1997; 127:289-93.

33 Iseman MD, Madsen LA. Chronic tuberculous empyema with bronchopleural fistula resulting in treatment failure and progressive drug resistance. Chest 1991; 100:124-7

34 Elliott $\mathrm{AM}$, Berning SE, Iseman MD, et al. Failure of drug penetration and acquisition of drug resistance in chronic tuberculous empyema. Tubercle Lung Dis 1995:76:463-7.

35 Schoeman JF, Morkel A, Seifart HI, et al. Massive posterior fossa tuberculous abscess developing in a young child treated for miliary tuberculosis. Possible role of very rapid acetylation of isoniazid. Pediatr Neurosurg 1998;29:64-8. 